\title{
Trombofilia en México
}

Benjamín Moncada1, Guillermo Ruiz-Argüelles² y Olga Johnson-Ponce ${ }^{1}$

${ }^{1}$ Universidad Autónoma de San Luis Potosi, Hospital Central Dr. Ignacio Morones Prieto, San Luis Potosí, S.L.P.; ${ }^{2}$ Centro de Hematologia y Medicina Interna de Puebla, Pue. México

Entre las anomalías genéticas que se traducen en enfermedad importante se encuentra la trombofilia primaria. En esta enfermedad se lleva a cabo la coagulación cuando en realidad no es necesaria. Trombofilia es lo opuesto a hemofilia, en la cual los pacientes sufren un sangrado excesivo aun ante lesiones mínimas, lo que no sucede en sujetos normales por estar intactos los mecanismos de la coagulación. La explicación de la hemofilia se reduce a un puñado de situaciones, mientras que en el caso de la trombofilia habría más de una docena de explicaciones. El conocimiento de estas dos enfermedades, tanto por el público como por la clase médica, es contrastante en favor de la hemofilia. Incluso en esta enfermedad se dispone de los medios terapéuticos para evitar un sangrado excesivo e incluso mortal ante la presunción de un problema como sería una cirugía mayor, o de carácter menor como una extracción dental. En la Secretaría de Salud estatal existen casos registrados de hemofilia a quienes se les apoya, mientras que no se tiene un apartado para la trombofilia.

El Dr. J. Guillermo Ruiz Argüelles ha estado dedicado a la búsqueda de la(s) alteración(es) que sean la causa de la trombofilia y se conoce así el perfil de este problema en nuestro país. Nosotros, desde hace 17 años, hemos estado investigando la clase de alteración que un paciente con trombofilia primaria presenta, apoyados en Clínica Ruiz, Laboratorios, de la ciudad de Puebla, a cargo del mencionado Dr. Ruiz-Argüelles (Tabla 1).

La indicación de la búsqueda ocurre cuando hay algunas de las siguientes circunstancias:
- Trombosis en una persona joven, alrededor de los 40 años de edad.

- Trombosis en sitios raros, no habituales para las trombosis.

- Mujeres que han sufrido una o más pérdidas fetales.

- Trombosis a pesar de estar recibiendo anti-coagulantes.

- Antecedentes familiares de trombosis.

- Trombosis recurrente sin factor precipitante aparente.

Como se aprecia en la Tabla 2, la explicación de la trombofilia no es única, sino que los fenómenos trombóticos pueden ser de distintos orígenes. Se aprecia también que la gran mayoría de los pacientes tienen más de una alteración en estos exámenes de laboratorio. La edad promedio de los pacientes en este grupo fue 30 años. Destacan a la cabeza la presencia de la mutación 677C-T del gen de la metilentetrahidrofolato reductasa, y también la alteración en la adhesividad plaquetaria, constituyendo esto último el síndrome de las plaquetas pegajosas (SPP). En menor proporción se encuentran el aumento del inhibidor del activador del plasminógeno, la mutación 20210GA del gen de la protrombina, los anticuerpos antifosfolípidos y la resistencia a la proteína C activada (Tabla 3).

Aparentemente, el sitio de la obstrucción trombótica se establece al azar, ya que no hay una preferencia por cierto lugar en función de la anomalía encontrada. Es posible que en el futuro se pueda aclarar este concepto, quizá por la actividad de receptores celulares o por un microambiente de moléculas como

\section{Correspondencia:}


Tabla 1. Elementos que constituyen el perfil de trombofilia en esta serie

Lipoproteína

Homocisteína

Fibrinógeno funcional

Agregación de fibrina

Plasminógeno

Inhibidor del activador del plasminógeno

Adhesividad plaquetaria

Antitrombina III

Anticoagulantes circulantes

Anticuerpos antifosfolípido

Anticardiolipina IgG e IgM

Anticuerpos anti-beta-2 glucoproteína

Proteína S de la coagulación

Proteína C de la coagulación

Resistencia a la proteína $\mathrm{C}$ activada

Mutaciones:

20210 G-A de la protrombina

677 C-T de la metilentetrahidrofolato reductasa

Factor V Leiden, Liverpool, Hong Kong, Cambridge

Haplotipo R2 del factor $V$

las citocinas y otros elementos afines que desempeñen un papel en determinar el sitio de la obstrucción. En la trombofilia todos los vasos sanguíneos pueden ser afectados, tanto arterias como venas y arteriolas.

Como se puede observar, no hay un solo varón en nuestra serie, lo que seguramente se debe a que ante una anomalía de fondo hay factores contribuyentes, y uno de ellos, el más importante, parecen ser las hormonas estrogénicas.

Publicaciones en distintos lugares del mundo han considerado a la mutación 677 de la MTHFR como la causa de la enfermedad trombótica'. Aunque encabeza la lista de las alteraciones en pacientes con trombosis $(62 \%$ en esta serie y $67 \%$ en la del Dr. Ruiz Arguelles), en nuestro medio se ha considerado un factor "débil» para ese propósito y que casi siempre se acompaña de otras alteraciones ${ }^{2}$. En nuestra serie se observa que solo en un paciente de los 27 estudiados la mutación 677 de la MTHFR fue la única alteración encontrada. Es interesante destacar que, en estudios con sujetos sanos en población mestiza en este país, el porcentaje de presencia de la mutación 677 alcanza la cifra del $78 \%$ 2.
Desde ese punto de vista se percibe entonces que la alteración más frecuente e importante sería el SPP. El hallazgo de esta alteración fue hecho por Mammen en 1988 y una década después anota sus hallazgos clínicos en relación a la alteración de laboratorio ${ }^{3}$, estableciéndose que la trombosis puede ocurrir en cualquier sitio de la economía corporal. No existe un marcador de laboratorio para hacer el diagnóstico del síndrome, aunque se han hecho intentos por llegar a ello 4 . De momento es una prueba «funcional» en la que se confrontan las plaquetas a dosis decrecientes de epinefrina y también de difosfato de adenosina (ADP), y se comparan con estándares normales; como el examen tiene que hacerse inmediatamente después de obtener la muestra de sangre, esto representa un inconveniente para un paciente en estado grave por no haber al alcance un laboratorio apropiado. En la práctica diaria este examen se realiza una vez que el paciente se recupera de su problema agudo, como en el caso de las trombosis en territorios críticos como el cerebro y el pulmón. Siendo el SPP frecuente y potencialmente grave por los sitios que pueden ser afectados, es importante crear conciencia del mismo $0^{6}$ y ahondar en su patogenia ${ }^{5}$ y en los aspectos terapéuticos ${ }^{7,8}$.

El defecto en el inhibidor del activador del plasminógeno ocupa un lugar intermedio en esta serie y ya se reconoce que es causa de trombofilia en distintos reportes ${ }^{9}$. Uno de nuestros casos (caso 13) combina esa alteración con el SPP10.

Con mucha frecuencia en la práctica clínica habitual se considera que una persona que tiene una trombosis sin explicación aparente tiene síndrome de anticuerpos antifosfolípidos sin considerar otras posibilidades. Por supuesto esto no redunda en el beneficio del paciente en cuanto a tratamiento y recuperación. En nuestros casos, la presencia de anticuerpos antifosfolípidos ocupa un lugar muy bajo.

Es también costumbre en nuestro medio, al pensar en trombofilia, considerar como factor causal una mutación del factor $V$ de la coagulación. Aunque esto es posible, entre nosotros esta alteración se observa muy poco, y lo mismo sucede con otras mutaciones del factor V (Cambridge, Hong Kong, Liverpool) ${ }^{11}$; en otros países, sin embargo, sobre todo en Europa, si es prevalente la presencia de mutaciones del factor $\mathrm{V}$.

También es frecuente en casos de trombofilia en nuestro medio pedir de manera prioritaria o exclusiva una determinación de los valores de las proteínas $C$ y $S$ de la coagulación, sobre todo de la primera. De 
Gaceta Médica de México. 2017;153

Tabla 2. Lista con los resultados obtenidos y los diagnósticos clínicos que motivaron la investigación

\begin{tabular}{|c|c|c|c|c|c|}
\hline N..$^{\circ}$ & Sexo & $\begin{array}{l}\text { Edad } \\
\text { (años) }\end{array}$ & Diagnóstico & Sitio afectado & Hallazgos \\
\hline 1 & $\mathrm{~F}$ & 28 & $\begin{array}{l}\text { Insuficiencia vascular } \\
\text { cerebral transitoria } \\
\text { Papulosis atrófica } \\
\text { maligna (enfermedad de } \\
\text { Degos) }\end{array}$ & $\begin{array}{l}\text { Cerebro } \\
\text { Piel }\end{array}$ & $\begin{array}{l}\text { Mutación } 677 \text { C-T de la } \\
\text { metilentetrahidrofolato reductasa } \\
\text { Mutación } 20210 \text { G-A de la protrombina } \\
\text { Resistencia a la proteína C activada }\end{array}$ \\
\hline 2 & $\mathrm{M}$ & $\mathrm{RN}$ & $\begin{array}{l}\text { Sano } \\
\text { Hijo de paciente } 1\end{array}$ & & $\begin{array}{l}\text { Mutación } 677 \text { C-T de la } \\
\text { metilentetrahidrofolato reductasa } \\
\text { Mutación } 20210 \text { G-A de la protrombina }\end{array}$ \\
\hline 3 & $\mathrm{~F}$ & 38 & $\begin{array}{l}\text { Livedo reticular } \\
\text { Insuficiencia vascular } \\
\text { transitoria }\end{array}$ & $\begin{array}{l}\text { Piel } \\
\text { Cerebro }\end{array}$ & $\begin{array}{l}\text { Mutación } 677 \text { C-T de la } \\
\text { metilentetrahidrofolato reductasa } \\
\text { SPP }\end{array}$ \\
\hline 4 & $\mathrm{~F}$ & 22 & $\begin{array}{l}\text { Embarazo normal } \\
\text { Sobrina paciente } 3\end{array}$ & & SPP \\
\hline 5 & $\mathrm{~F}$ & 24 & Trombosis cerebral media & Cerebro & $\begin{array}{l}\text { Mutación } 677 \text { C-T de la } \\
\text { metilentetrahidrofolato reductasa } \\
\text { SPP }\end{array}$ \\
\hline 6 & $\mathrm{~F}$ & 40 & $\begin{array}{l}\text { Trombosis mesentérica } \\
\text { TEP } \\
\text { Pérdidas fetales }\end{array}$ & $\begin{array}{l}\text { Intestino y pulmón } \\
\text { Placenta } \\
\text { Piernas }\end{array}$ & SPP \\
\hline 7 & $\mathrm{~F}$ & 31 & Trombosis venosa profunda & Pierna & SPP \\
\hline 8 & $\mathrm{~F}$ & 30 & $\begin{array}{l}\text { TEP } \\
\text { Insuficiencia vascular } \\
\text { cerebral transitoria }\end{array}$ & $\begin{array}{l}\text { Pierna } \\
\text { Pulmón } \\
\text { Cerebro }\end{array}$ & $\begin{array}{l}\text { Mutación } 677 \text { C-T de la } \\
\text { metilentetrahidrofolato reductasa } \\
\text { SPP } \\
\text { Mutación } 20210 \text { G-A de la protrombina } \\
\text { IAP }\end{array}$ \\
\hline 9 & $\mathrm{~F}$ & 17 & $\begin{array}{l}\text { Parto normal } \\
\text { Hija paciente } 8\end{array}$ & Placenta & $\begin{array}{l}\text { SPP } \\
\text { Mutación } 20210 \text { G-A de la protrombina }\end{array}$ \\
\hline 10 & $\mathrm{~F}$ & 17 & $\begin{array}{l}\text { Eritema nudoso } \\
\text { Parto prematuro }\end{array}$ & Piel y placenta & $\begin{array}{l}\text { SPP } \\
\text { Mutación } 20210 \text { G-A de la protrombina } \\
\text { IAP }\end{array}$ \\
\hline 11 & $\mathrm{~F}$ & 34 & Oclusión seno longitudinal & Cerebro & SPP \\
\hline 12 & $\mathrm{~F}$ & 48 & Atrofia blanca & $\begin{array}{l}\text { Piel } \\
\text { Pulmón }\end{array}$ & $\begin{array}{l}\text { Mutación } 677 \text { C-T de la } \\
\text { metilentetrahidrofolato reductasa } \\
\text { Mutación } 20210 \text { G-A de la protrombina }\end{array}$ \\
\hline 13 & $\mathrm{~F}$ & 12 & $\begin{array}{l}\text { Atrofia blanca } \\
\text { Vasculopatía livedoide }\end{array}$ & Piel & $\begin{array}{l}\text { SPS } \\
\text { IAP }\end{array}$ \\
\hline 14 & $\mathrm{~F}$ & 43 & $\begin{array}{l}\text { Piel } \\
\text { Atrofia blanca } \\
\text { Trombosis venosas previas }\end{array}$ & $\begin{array}{l}\text { Piel } \\
\text { Venas piernas }\end{array}$ & $\begin{array}{l}\text { SPS } \\
\text { Anticuerpos antifosfolípidos } \\
\text { Anti-beta-2 glucoproteína }\end{array}$ \\
\hline 15 & $\mathrm{~F}$ & 37 & TEP posaborto & $\begin{array}{l}\text { Venas pierna } \\
\text { Pulmón }\end{array}$ & $\begin{array}{l}\text { SPP } \\
\text { IAP }\end{array}$ \\
\hline 16 & $\mathrm{~F}$ & 40 & Síndrome de Sneddon & Piel y cerebro & $\begin{array}{l}\text { Antitrombina (1\%) } \\
\text { Proteína C (8) } \\
\text { Leiden (6) } \\
\text { Homocisteína (1\%) }\end{array}$ \\
\hline 17 & $F$ & 33 & $\begin{array}{l}\text { Parto prematuro } \\
\text { Abuela y madre con } \\
\text { preeclampsia }\end{array}$ & Placenta & $\begin{array}{l}\text { SPP } \\
\text { IAP }\end{array}$ \\
\hline
\end{tabular}


Tabla 2. Lista con los resultados obtenidos y los diagnósticos clínicos que motivaron la investigación (Continued)

\begin{tabular}{|c|c|c|c|c|c|}
\hline N. ${ }^{\circ}$ & Sexo & $\begin{array}{l}\text { Edad } \\
\text { (años) }\end{array}$ & Diagnóstico & Sitio afectado & Hallazgos \\
\hline 18 & $\mathrm{~F}$ & 13 & Tromboflebitis & Venas piernas & $\begin{array}{l}\text { SPP } \\
\text { Anticoagulantes circulantes } \\
\text { Anticuerpos anti-beta-2 glucoproteína } \\
\text { Anticuerpos anticardiolipina } \\
\text { Mutación } 677 \text { C-T de la } \\
\text { metilentetrahidrofolato reductasa }\end{array}$ \\
\hline 19 & $\mathrm{~F}$ & 40 & Trombosis arteria cerebelosa & Cerebro & $\begin{array}{l}\text { IAP } \\
\text { Mutación } 677 \text { C-T de la } \\
\text { metilentetrahidrofolato reductasa } \\
\text { Fibrinógeno elevado }\end{array}$ \\
\hline 20 & $\mathrm{~F}$ & 38 & $\begin{array}{l}\text { Insuficiencia vascular } \\
\text { transitoria }\end{array}$ & Cerebro & IAP \\
\hline 21 & $\mathrm{~F}$ & 40 & Atrofia blanca & Piel & $\begin{array}{l}\text { Mutación } 677 \text { C-T de la } \\
\text { metilentetrahidrofolato reductasa } \\
\text { Mutación } 20210 \text { G-A de la protrombina }\end{array}$ \\
\hline 22 & $\mathrm{~F}$ & 26 & Trombosis seno longitudinal & Cerebro & $\begin{array}{l}\text { IAP } \\
\text { Mutación } 677 \text { C-T de la } \\
\text { metilentetrahidrofolato reductasa } \\
\text { DM } \\
\text { Hipertrigliceridemia }\end{array}$ \\
\hline 23 & $\mathrm{~F}$ & 40 & TEP & $\begin{array}{l}\text { Pulmón } \\
\text { Venas piernas }\end{array}$ & $\begin{array}{l}\text { Mutación } 677 \text { C-T de la } \\
\text { metilentetrahidrofolato reductasa }\end{array}$ \\
\hline 24 & $\mathrm{~F}$ & 21 & Anetoderma & Piel & $\begin{array}{l}\text { SPP } \\
\text { Mutación } 677 \text { C-T de la } \\
\text { metilentetrahidrofolato reductasa }\end{array}$ \\
\hline 25 & $\mathrm{~F}$ & 34 & Trombosis cerebral media & Cerebro & $\begin{array}{l}\text { SPP } \\
\text { Mutación } 677 \text { C-T de la } \\
\text { metilentetrahidrofolato reductasa } \\
\text { Anticuerpos antifosfolípidos }\end{array}$ \\
\hline 26 & $\mathrm{~F}$ & 41 & $\begin{array}{l}\text { Atrofia blanca. } \\
\text { Distrofia muscular progresiva } \\
\text { escápulo-humeral }\end{array}$ & Piel & $\begin{array}{l}\text { Mutación } 677 \text { C-T de la } \\
\text { metilentetrahidrofolato reductasa } \\
\text { Fibrinógeno y agregación de fibrina } \\
\text { elevados }\end{array}$ \\
\hline 27 & $\mathrm{~F}$ & 23 & $\begin{array}{l}\text { Abortos } \\
\text { Partos prematuros } \\
\text { Eclampsia } \\
\text { Trombosis piernas }\end{array}$ & $\begin{array}{l}\text { Placenta } \\
\text { Venas piernas }\end{array}$ & $\begin{array}{l}\text { Anticuerpos antifosfolípidos } \\
\text { Anticuerpos anti-beta-2 glucoproteína } \\
\text { Mutación } 677 \text { C-T de la } \\
\text { metilentetrahidrofolato reductasa }\end{array}$ \\
\hline
\end{tabular}

IAP: inhibidor del activador del plasminógeno; DM: diabetes mellitus; F: femenino; M: masculino; SPP: síndrome de las plaquetas pegajosas; TEP: tromboembolia pulmonar.

nuevo esta alteración es de bajo perfil entre nosotros.

En los casos raros que se presentan, suele haber una gran variedad de las manifestaciones ${ }^{12}$.

Lo aquí relatado tiene gran importancia desde diferentes ángulos:

- Reconocer que existe la posibilidad técnica para precisar el problema que origina un estado de trombofilia primaria, merced a los esfuerzos del grupo de trabajo del Dr. Ruiz Argüelles.

- Establecer el carácter regional de estas alteraciones, y de esa manera no seguir modelos de investigación operantes en otros países, sino buscar preferentemente las anomalías que se presentan entre nosotros.

- Apreciar el papel de los estrógenos en esta enfermedad. El ejemplo del caso 13 ilustra este punto: paciente sana toda su niñez, que a los 12 años tiene oclusión arteriolar dando el síndrome de atrofia blanca/vasculopatía livedoide justo cuando empieza la adolescencia (Fig. 1). También mujeres en quienes ocurren las trombosis durante el embarazo (casos 15, 17 y 27).

- Creación de criterios preventivos para tratar de impedir la recurrencia de la trombosis en las 

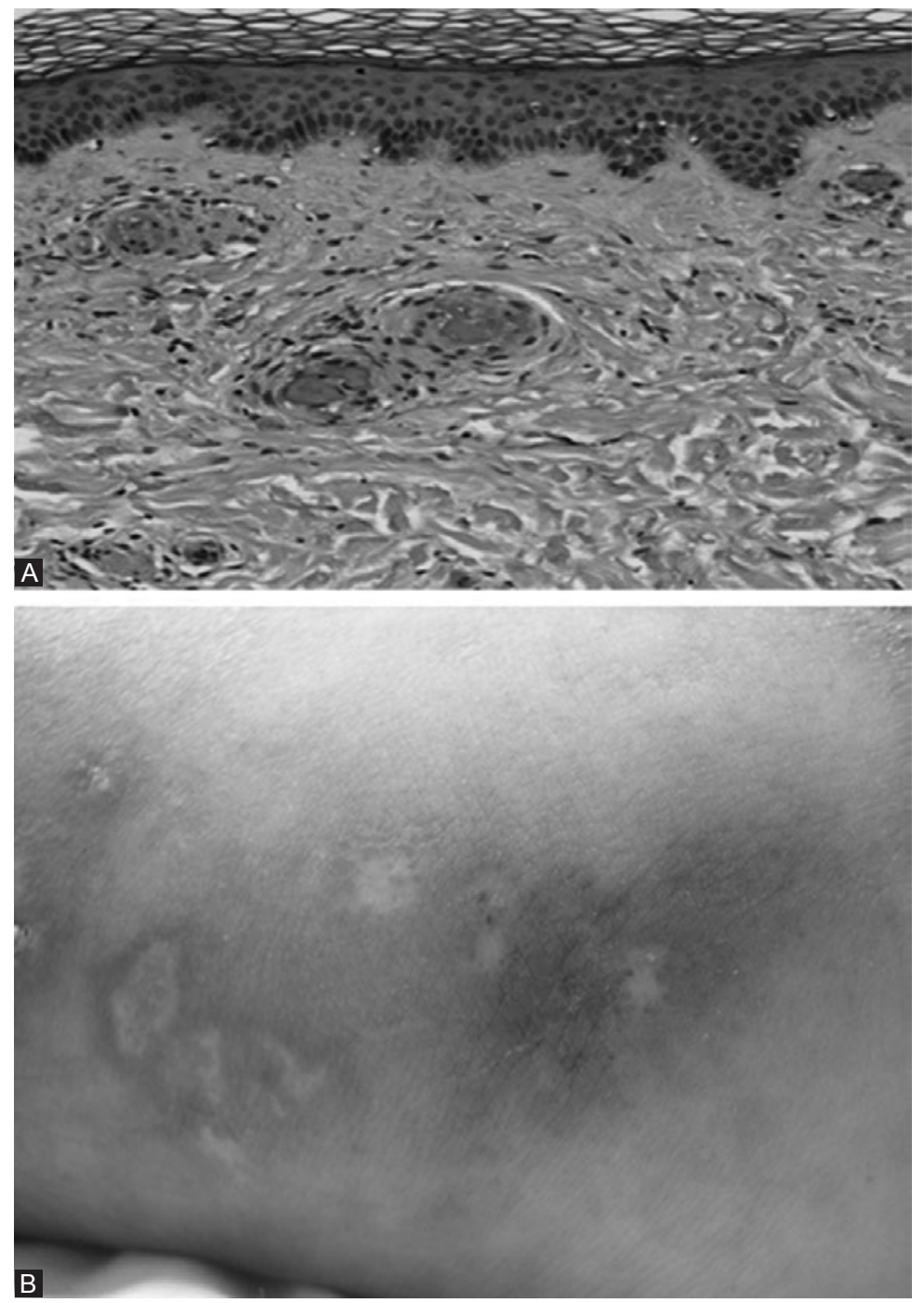

Figura 1. A: biopsia del paciente 13 con oclusión arteriolar de vasos del pie sin inflamación. B: lesiones cutáneas, activas y cicatrizadas.

Tabla 3. Alteraciones presentadas en 27 pacientes estudiados

\begin{tabular}{lcc}
\hline & $\mathbf{n}$ & $\%$ \\
\hline $\begin{array}{l}\text { Mutación del gen 667 C-T de la } \\
\text { metilentetrahidrofolato reductasa }\end{array}$ & 17 & 32.1 \\
Incremento en la actividad plaquetaria & 16 & 30.2 \\
Incremento en el inhibidor del activador del & 8 & 15.1 \\
plasminógeno & & \\
Mutación del gen 20210 G-A de la protrombina & 7 & 13.2 \\
Anticuerpos antifosfolípidos & 3 & 5.7 \\
Aumento del fibrinógeno activado & 1 & 1.9 \\
Aumento en la agregación de fibrina & 1 & 1.9 \\
Total & $\mathbf{5 3}$ & $\mathbf{1 0 0}$ \\
\hline
\end{tabular}

personas afectadas, e impedir que esto ocurra en familiares cercanos, buscando en ellos específicamente las anomalías del familiar enfermo. Lo anterior queda ilustrado en los casos 4 y 17.
En el primero, la sobrina sana de la paciente 3 al iniciar embarazo se detectó que compartía con la tía el SPP, y con ello tuvo un embarazo vigilado que terminó con éxito. Algo similar sucedió con el caso 17, que por patología sugestiva de patogenia trombótica en la madre y la abuela en el pasado remoto, se realizó el perfil de trombofilia y se encontró el SPP y un aumento del inhibidor del activador del plasminógeno, por lo que en ella también se tuvieron cuidados especiales, terminando con el embarazo a las 36 semanas viendo que se iniciaba la trombosis en la placenta (Fig. 2).

Como en todo conocimiento nuevo en algunas prácticas, la médica en particular, suele haber ignorancia, no aceptación o rechazo del mismo, con lo que se evitaría la atención apropiada de los enfermos. Esto se hace evidente en los casos 14 y 16 . En el primero, la paciente con atrofia blanca y trombosis venosas 


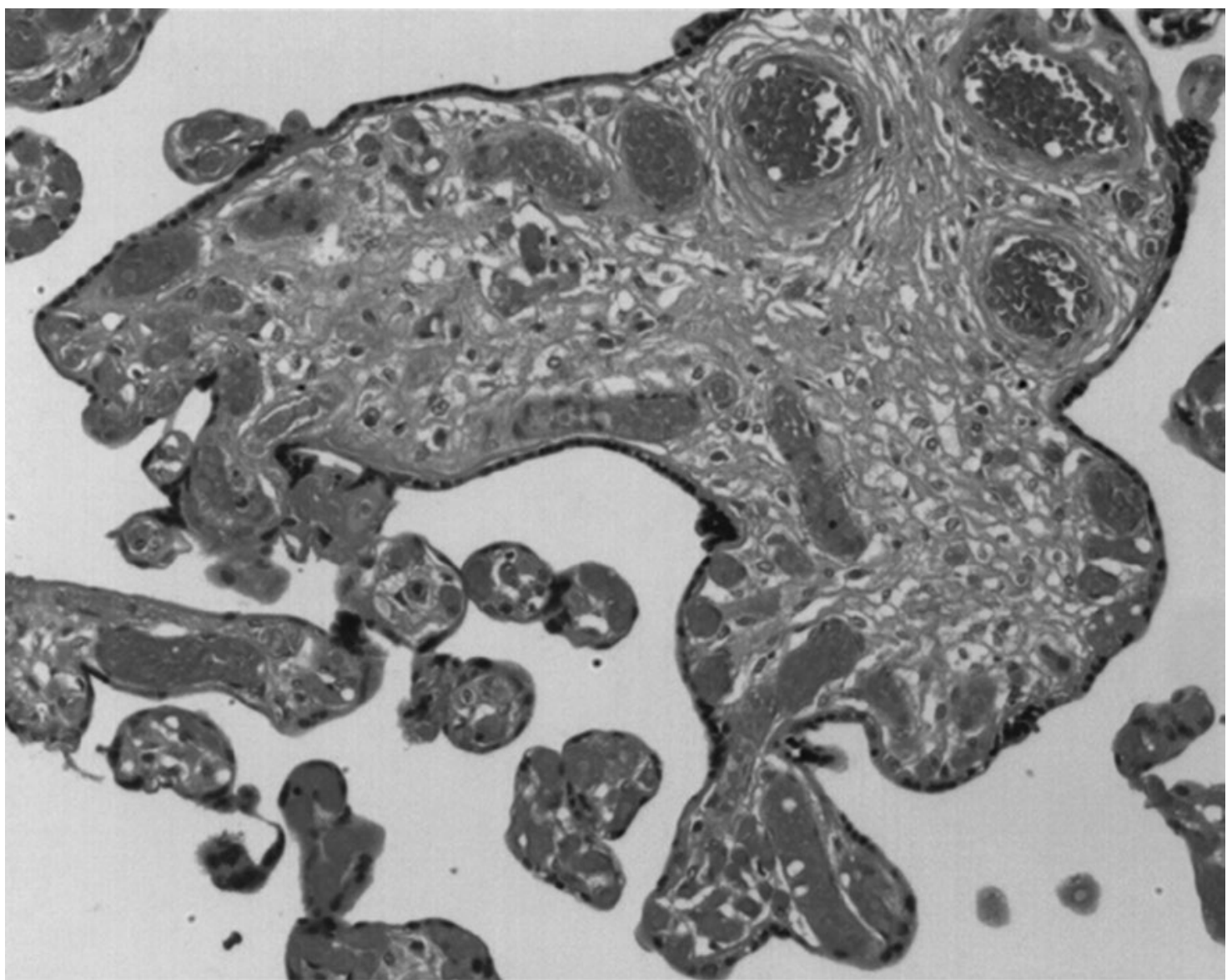

Figura 2. Placenta de la paciente de 33 años del caso 17 en quien se interrumpió el embarazo a las 36 semanas por presentar sufrimiento fetal. Se aprecia en la foto que se inician las trombosis de los vasos placentarios.

previas tenía una hermana con edad mayor de 40 años y un primer embarazo; se le sugirió a la persona encargada de su atención ginecológica que realizara los exámenes que su hermana tenía positivos para dar cuidados especiales en el curso de su embarazo. Esta recomendación no fue tomada en cuenta y se corrieron riesgos innecesarios en ella. En el caso 16, con síndrome de Sneddon, que consiste en oclusiones de las arterias de las piernas y también del cerebro, se aceptó la recomendación de realizar el perfil de trombofilia, pero solo se hizo de manera parcial; cuatro estudios, dos de ellos que ocupan menos del $1 \%$ en la experiencia mexicana y otros dos que ocupan los lugares 8 y 6 en nuestro país. La razón: solo aceptó realizar las pruebas que se hacían en la institución de salud a la que pertenecía.

El tratamiento de la trombofilia en los episodios agudos va a depender del sitio afectado y de la intervención de los especialistas que amerite la región afectada. Pero de acuerdo con la alteración en cuestión habrá necesidad de uno u otro fármaco de manera temporal o indefinida, y también medidas generales y de sentido común, como evitar la inmovilidad, por ejemplo durante viajes largos, evitar la deshidratación, y evitar medicamentos innecesarios o productos "naturistas" que pudieran ser adyuvantes en el proceso de trombosis.

Un conocimiento reciente ha sido que el estrés influye en las trombosis ${ }^{13}$, por lo que sería altamente recomendable aconsejar a los pacientes en este sentido.

\section{Bibliografía}

1. Ali Z, Troncoso JC, Fowler DR. Recurrent cerebral venous thrombosis associated with heterozygote methylenetetrahydrofolate reductase C677T mutation and sickle cell trait without homocysteinemia. An autopsy case report and review of literature. Forensic Sci Int. 2014;242:e52-5.

2. Ruiz-Delgado G, Ruiz Argüelles GJ. Estudios en México sobre el gen MTHFR. Salud Pública de México. 2008:50:5

3. Mammen EF. Sticky platelet syndrome. Semin Thromb Hemostat. 1999;25:361-5.

4. Ruiz Argüelles GJ, Garces Eisele J, Camacho-Alarcón C, et al. Primary thrombophilia in Mexico IX. Primary thrombophilia in Mexico IX: the glycoprotein IIla PLA1/A2 polymorphism is not associated with the sticky platelet syndrome phenotype. Clin Appl Thromb Hemost. 2013; 19:689-92.

5. Ruiz-Argüelles GJ, López-Martínez B, Cruz-Cruz D, et al. Primary thrombophilia in Mexico III. A prospective study of the sticky platelet sydrome. Clin Appl Thromb Hemost. 2002;8:T1-T5.

6. Moncada B, Ruiz-Argüelles GJ, Castillo-Martínez C. The sticky platelet syndrome. Hematology. 2013;18:230-2. 
7. Velázquez-Sánchez-de Cima S, Zamora-Ortiz G, Hernández-Reyes J, et al. Primary thrombophilia in Mexico X. A prospective study of the treatment of the sticky platelet síndrome. Clinical and Applied Thrombosis/hemostasis.2015;21:91-5.

8. Kubisz P, Stasko J, Holly P. Sticky platelet syndrome. Semin Thromb Hemost. 2013;39:674-83.

9. Agirbasli M, Eren M, Eren F, et al. Enhanced functional stability of plasminogen activator inhibitor-1 in patients with livedoid vasculopathy. J Thromb Thrombolysis. 2011;32:59-63.

10. Castillo-Martínez C, Moncada B, Valdés-Rodríguez R, et al. Livedoid vasculopathy (LV) associated with sticky platelets síndrome type 3 and enhanced activity of plasminogen activator inhibitor (PAI-1) anomalies. Int J Dermatol. 2014;53:1495-7.

11. Ruiz-Argüelles GJ, Poblete Naredo I, Reyes Nuñez V, et al. Primary thrombophilia in Mexico IV. Frequency of the Leiden, Cambridge, Hong Kong, Liverpool and HR2 haplotype polymorphisms in the factor $\mathrm{V}$ gene of a group of thrombophilic Mexican Mestizo patients. Rev Invest Clin. 2004;56:600-4.

12. De Lemus Varela ML, Arriaga-Dávila J de J, Salinas-Lopez MP. Protein C congenital deficiency. A case report. Gac Med Mex. 2005;141:229-31.

13. Surtees PG Wainwright NW, Luben NR, et al. Psychologial distress, major depressive disorders, and risk of stroke. Neurology. 2008;70:788-94. 